
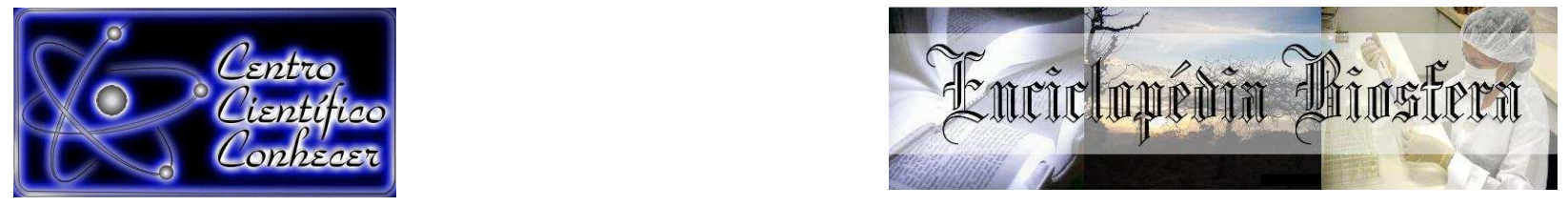

\title{
TAXAS DE RECRUTAMENTO E MORTALIDADE DE ESPÉCIES DE LECYTHIDACEAE APÓS COLHEITA FLORESTAL NA AMAZÔNIA ORIENTAL
}

Pamella Carolline Marques dos Reis Reis ${ }^{1^{*}}$, Leonardo Pequeno Reis ${ }^{1}$, Agostinho Lopes de Souza ${ }^{2}$, Lyvia Julienne Sousa Rego ${ }^{1}$, Liniker Fernandes da Silva ${ }^{5}$

${ }^{1}$ Doutorando (a) do Programa de Pós-graduação em Ciência Florestal, Departamento de Engenharia Florestal, Universidade Federal de Viçosa, Viçosa, MG, Brasil. (pamella.reis@ufv.br)

${ }^{2}$ Professor do Programa de Pós-graduação em Ciência Florestal, Departamento de Engenharia Florestal, Universidade Federal de Viçosa, Viçosa, MG, Brasil.

${ }^{3}$ Professor adjunto I da Universidade Federal do Recôncavo da Bahia, Cruz das Almas, BA, Brasil.

Recebido em: 03/10/2016 - Aprovado em: 21/11/2016 - Publicado em: 05/12/2016 DOI: 10.18677/EnciBio_2016B_092

\section{RESUMO}

O monitoramento de florestas colhidas seletivamente é de grande importância para conhecer o comportamento das espécies. Entender os processos dinâmicos como o recrutamento e a mortalidade são fundamentais para prescrever tratamentos silviculturais adequados para as espécies remanescentes. O objetivo do trabalho foi avaliar o efeito da colheita seletiva de madeirais na dinâmica do recrutamento e mortalidade das espécies de Lecythidaceae. Foram avaliados nesse estudo os parâmetros recrutamento $\left(\% \mathrm{ano}^{-1}\right)$ e mortalidade $\left(\% \mathrm{ano}^{-1}\right)$ de árvores de Lecythidaceae após 13 da colheita de madeiras. A área de estudo fica localizada no município de Moju, estado do Pará. Em 1995 foram instaladas 22 parcelas permanentes com dimensão de $50 \times 100 \mathrm{~m}$ totalizando 11 ha de amostragem. Onde foram medidas todas as árvores com $D A P \geq 10 \mathrm{~cm}$ e foi determinado o nome vulgar. Em 1997 ocorreu a colheita, e foi retirado um volume de $23 \mathrm{~m}^{3} \mathrm{ha}^{-1}$. Foram realizadas três medições nas parcelas, 1995 antes da colheita e as remedições póscolheita em 1998 e 2010. Analisando todo o período de monitoramento (1995 a 2010) o recrutamento $\left(1,1 \%\right.$ ano $\left.^{-1}\right)$ foi maior que a mortalidade $\left(1,0 \% \mathrm{ano}^{-1}\right)$, avaliando o primeiro período de 1995 a 1998 , a mortalidade $\left(2,0 \%\right.$ ano $\left.^{-1}\right)$ foi maior que o recrutamento $\left(1,9 \%\right.$ ano $\left.^{-1}\right)$, devido aos impactos da colheita. Entretanto, analisando o segundo período de 1998 a 2010 o recrutamento $\left(0,9 \%\right.$ ano $\left.{ }^{-1}\right)$ ultrapassou a mortalidade $\left(0,8 \% \mathrm{ano}^{-1}\right)$, demonstrando que a floresta começou a se recuperar. A colheita florestal teve impacto positivo na estrutura das populações das espécies de Lecythidaceae estudadas.

PALAVRAS-CHAVE: exploração de impacto reduzido, manejo florestal, matá-matá.

\section{RECRUITMENT AND MORTALITY RATES OF LECYTHIDACEAE SPECIES AFTER FOREST HARVEST IN EASTERN AMAZON}

\begin{abstract}
The monitoring of forests selectively harvested is of great importance to know the behavior of the species. Understanding the dynamic processes such as recruitment 2016
\end{abstract} ENCICLOPÉDIA BIOSFERA, Centro Científico Conhecer - Goiânia, v.13 n.24; p.970 
and mortality are essential to prescribe silvicultural treatments suitable for the remaining species. The aim of the study was to evaluate the effect of wood selective harvest in the dynamics of recruitment and mortality of Lecythidaceae species. The parameters recruitment ( $\%$ year $\left.^{-1}\right)$ and mortality ( $\%$ year $\left.^{-1}\right)$ of Lecythidaceae trees after 13 years of wood harvest were evaluated in this study. The study area is located in the city of Moju, Pará state. In 1995, 22 permanent plots with size of $50 \mathrm{x}$ $100 \mathrm{~m}$ were installed totaling 11 ha of sampling. On which all trees with DAP $\geq 10 \mathrm{~cm}$ were measured and the common name was given. In 1997 the harvest occurred and a volume of $23 \mathrm{~m}^{3} \mathrm{~h}^{-1}$ was harvested. Three measurements were made in the plots, before harvest in 1995 and post-harvest remesuraments in 1998 and 2010. Analyzing the entire monitoring period (1995 to 2010), recruitment (1.1\% year ${ }^{-1}$ ) was higher than mortality $\left(1.0 \%\right.$ year $\left.^{-1}\right)$, when assessed the first period 1995-1998, mortality $\left(2.0 \%\right.$ year $\left.^{-1}\right)$ was higher than recruitment $\left(1.9 \%\right.$ year $\left.^{-1}\right)$ due to harvest impacts. However, analyzing the second period $1998-2010$ recruitment $\left(0.9 \%\right.$ year $\left.^{-1}\right)$ exceeded the mortality $\left(0.8 \%\right.$ year $\left.^{-1}\right)$, demonstrating that the forest began to recover. The forest harvesting had a positive impact on the structure of the populations of the Lecythidaceae species studied.

KEYWORDS: Forest Management, Reduced Exploitation Impact, Mata mata.

\section{INTRODUÇÃO}

O monitoramento da recuperação das espécies após a colheita é fundamental para indicar ações de manejo visando sua conservação. Nesse contexto, o estudo das taxas de recrutamento e mortalidade de árvores são essenciais para entender a dinâmica das populações das espécies colhidas. Esses estudos também são a base para o desenvolvimento de modelos de crescimento e produção, que possibilitam indicar formas sustentáveis de manejo para produção de madeiras em florestas tropicais (ZHANG et al., 2012; NASCIMENTO et al., 2013; REIS et al., 2014).

A avaliação das taxas e processos de mortalidade e recrutamento de árvores contribui para o entendimento das perturbações decorridas por ações naturais e ou antrópicas, como apresentado em diferentes estudos em florestas tropicais (PUTZEL et al., 2011; TOLEDO, et al., 2012; YAMADA et al., 2013; DARRIGO et al., 2016). Essas taxas são indicativas da função do ecossistema (ROSSI et al., 2007a; ROSSI et al., 2007b). Nesse sentido, o sucesso do segundo corte está intimamente ligado à recomposição da densidade, que depende do balanço entre recrutamento e mortalidade. Se houver maior recrutamento das espécies comerciais, após distúrbios, maior será a regeneração econômica da floresta (REIS et al., 2014).

Estudos que avaliam as taxas de recrutamento e mortalidade especificamente para um grupo de espécies e individualizado por espécie de elevado valor ecológico e econômico, oferecem subsídios para intervenções mais adequadas por apresentar, claramente por espécie, o comportamento individualizado do efeito da colheita, não correndo o risco de ser mais intensivo para uma determinada espécie, ficando na capacidade de suporte de regeneração, garantindo que a taxa de recrutamento seja maior que a taxa de mortalidade (REIS et al., 2014).

Portanto um grupo de espécies coma as Lecythidaceae possuem elevada importância econômica na Amazônia e tem grande representatividade no manejo florestal. Atualmente, no Estado do Pará, 28 espécies de Lecythidaceae são exploradas para produção de madeira (REIS et al., 2016). Dentre elas, nove são consideradas neste trabalho: três do gênero Eschweilera, três de Lecythis e de Couratari. Estudos sobre de dinâmica após a colheita são importantes para essas 
espécies, já que a maioria não possui informações ecológicas insuficientes para dar suporte à sua colheita de forma sustentável (REIS et al., 2014). O objetivo do trabalho foi avaliar a dinâmica de espécies de Lecythidaceae através das taxas de recrutamento e mortalidade após colheita seletiva de madeirais na Amazônia Oriental.

\section{MATERIAL E MÉTODOS}

O estudo foi conduzido em uma área de floresta localizada no município de

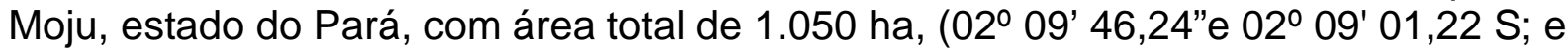

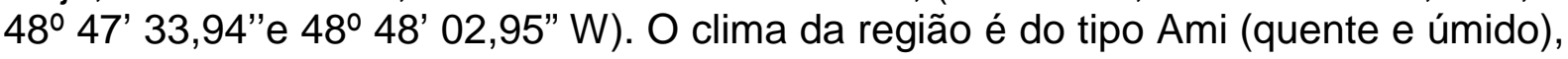
segundo a classificação de Köppen. A precipitação pluviométrica anual varia de 2.000 a $3.000 \mathrm{~mm}$. A umidade relativa do ar é de $85 \%$, com temperatura média anual de $26^{\circ} \mathrm{C}$. O relevo é plano, com formação de Latossolo Amarelo distrófico com diferentes texturas. A tipologia da área experimental é Floresta Ombrófila Densa de terra firme (REIS et al., 2013; REIS et al., 2015).

Em 1997 foi realizada uma colheita de madeiras em 200 ha, onde foram retiradas, em média, 3,3 árvores ha ${ }^{-1}$, correspondendo a um volume médio de $23 \mathrm{~m}^{3}$ ha $^{-1}$, de 25 espécies (REIS et al., 2015), considerando o Diâmetro Mínimo de Corte $(\mathrm{DMC})$ de $65 \mathrm{~cm}$. Couratari guianensis Aubl. foi a única espécie de Lecythidaceae que foi colhida, sendo extraídos, em média, 0,21 árvores ha-1 com volume de 1,84 $\mathrm{m}^{3}$ ha $^{-1}$ (REIS et al., 2014).

Em 1995, antes da colheita, foram implantadas, aleatoriamente, e medidas 22 parcelas permanentes com dimensões de $50 \times 100 \mathrm{~m}$, correspondendo a 11 ha de área amostral. Todas as árvores com Diâmetro à Altura do Peito (DAP) $\geq 10 \mathrm{~cm}$ foram registradas e medidas. As parcelas foram remedidas em 1998 e 2010 (REIS et al., 2015).

Para a identificação botânica das espécies, foram coletadas no mínimo cinco amostras de material botânico, por árvore, para cada nome vulgar de Lecythidaceae, totalizando 102 coletas. A determinação foi realizada por comparação com amostras botânicas existentes no herbário IAN da Embrapa Amazônia Oriental de acordo com REIS et al., (2014) e também com o auxílio da revisão taxonômica MORI \& PRANCE (1990).

Foram realizadas três medições nas parcelas, (1995) antes da colheita e as remedições pós-colheita (1998 e 2010). Para calcular a taxa de mortalidade (\% ano $\left.{ }^{1}\right)$ foi utilizada a equação de SHEIL et al. (1995):

$$
M=\left(1-\left(\left(N_{0}-m\right) / N_{0}\right)^{\frac{1}{t}}\right) \times 100
$$

Em que,

M: é a taxa de mortalidade anual;

m: é o número de árvores mortas, desconsiderando as árvores colhidas;

$\mathrm{N}_{0}$ : é o número de indivíduos da população inicial; e

t: é o tempo em anos.

Como causas da mortalidade foram consideradas as de origem natural e antrópica. De acordo com ROSSI et al. (2007) as causas da mortalidade natural são as ocasionadas por: ataques de patógenos; parasitas e herbívoros; tempestades; danos causados por chuvas fortes e morte por idade, considerando que todo ser vivo tem um período de vida finito. Já para mortalidade antrópica, foram considerados os danos ocasionados pela derruba e arraste das toras. 
Neste trabalho foi considerado como recrutamentos, as árvores que atingiram ou ultrapassaram o diâmetro mínimo de $10 \mathrm{~cm}$ em cada medição, a partir da segunda (REIS et al., 2013). Para o cálculo da taxa de recrutamento (\% ano ${ }^{-1}$ ) foi utilizada a equação de SHEIL \& MAY (1996):

$$
I=\left(1-\left(1-i / N_{t}\right)^{\frac{1}{\tau}}\right) \times 100
$$

Em que,

I: é a taxa de recrutamento anual;

$\mathrm{Nt}$ : é o número final de árvores sobreviventes depois de t;

i: é o número de recrutamentos; e

t: tempo em anos.

Para o processamento dos dados foi utilizado o software MFT (Monitoramentos de Florestas Tropicais) da Embrapa e o software Microsoft Excel 2010.

\section{RESULTADOS E DISCUSSÃO}

Foram encontradas na área de estudo 14 espécies da família Lecythidaceae: Eschweilera coriacea (DC.) S.A., Eschweilera grandiflora (Aubl.) Sandwith, Eschweilera amazonica R. Knuth, Eschweilera ovata (Cambess.) Mier, Eschweilera pedicellata (Rich.) S.A. Mori, Eschweilera nana (O. Berg) Miers, Eschweilera albiflora (DC.) Mier, Lecythis idatimon Aubl., Lecythis lurida (Mieônrs) S.A. Mori, Lecythis pisonis Cambess., Lecythis chartacea O. Berg, Couratari guianensis Aubl., Couratari oblongifolia Ducke \& R. Knuth e Couratari stellata A.C. Sm.

A comunidade total amostrada (11 ha), durante 15 anos (1995 a 2010) apresentou o recrutamento maior que a mortalidade (Tabela 1), com a taxa de recrutamentos de 2,4\% e a de mortalidade igual de1,9\%. A taxa de mortalidade atual encontra-se dentro do padrão esperado, de $1 \%$ a $2 \%$, para florestas tropicais segundo PHILLIPS et al. (1998).

TABELA 1. Taxas de mortalidade e recrutamento das espécies de Lecythidaceae com $\mathrm{DAP} \geq 10 \mathrm{~cm}$, de floresta, submetida à colheita de madeiras em 1997, na Amazônia.

\begin{tabular}{|c|c|c|c|c|c|c|}
\hline \multirow[t]{3}{*}{ Espécies } & \multicolumn{2}{|c|}{$1995-1998$} & \multicolumn{2}{|c|}{$1998-2010$} & \multicolumn{2}{|c|}{$1995-2010$} \\
\hline & Mortalidade & Recrutamento & Mortalidade & Recrutamento & Mortalidade & Recrutamento \\
\hline & $\%$ ano -1 & $\%$ ano -1 & $\%$ ano -1 & $\%$ ano -1 & $\%$ ano -1 & $\%$ ano -1 \\
\hline Eschweilera & & & & & & \\
\hline amazonica & 1,1 & 2,2 & 4,3 & 0,5 & 4,0 & 1,2 \\
\hline Couratari guianensis & 6,8 & 0,0 & 0,0 & 1,1 & 1,5 & 0,9 \\
\hline Lecythis pisonis & 1,9 & 0,0 & 1,1 & 0,0 & 1,3 & 0,0 \\
\hline $\begin{array}{l}\text { Lecythis idatimon } \\
\text { Eschweilera }\end{array}$ & 2,6 & 1,6 & 0,7 & 0,7 & 1,1 & 0,9 \\
\hline $\begin{array}{l}\text { coriacea } \\
\text { Eschweilera }\end{array}$ & 2,0 & 2,1 & 0,8 & 1,0 & 1,1 & 1,3 \\
\hline $\begin{array}{l}\text { grandiflora } \\
\text { Couratari }\end{array}$ & 0,0 & 2,9 & 0,2 & 1,1 & 0,2 & 1,6 \\
\hline oblongifolia & 0,0 & 0,0 & 0,0 & 0,0 & 0,0 & 0,0 \\
\hline Couratari stellata & 0,0 & 0,0 & 0,0 & 0,0 & 0,0 & 0,0 \\
\hline Eschweilera nana & 0,0 & 0,0 & 0,0 & 0,0 & 0,0 & 0,0 \\
\hline Eschweilera ovata & 0,0 & 0,0 & 0,0 & 1,1 & 0,0 & 0,9 \\
\hline
\end{tabular}


Eschweilera

pedicellata

Lecythis chartacea

Eschweilera albiflora

Lecythis lurida

Total Lecythidaceae

0,0

0,0

0,0

0,0

0,0

0,0

Total-comunidade

0,0

0,0

0,0

0,0

0,0

As espécies da família Lecythidaceae apresentaram balanço positivo, ou seja, recrutamento $(1,1 \%)$ maior que a mortalidade $(1,0 \%)$, quando considerado todo 0 período de avaliação (Tabela 1). De acordo com ROSSI et al. (2007) os danos originados por interferências humanas também podem contribuir com a mortalidade. A derrubada de árvores pode esmagar árvores menores, as máquinas podem danificar raízes e cascas e criar pontos de entrada para doenças e podridão. Entretanto, embora a floresta natural esteja em dinâmica contínua, há um equilíbrio, de forma que as árvores mortas são continuamente substituídas por novos indivíduos.

Segundo REIS et al. (2014) a abertura do dossel após a colheita florestal nessa área favoreceu o recrutamento de novos indivíduos da família Lecythidaceae. Demonstrando que o aumento da radiação solar proporcionado pela abertura do dossel aumentou o crescimento das árvores e, consequentemente, aumentou a densidade e a área basal das árvores remanescentes.

No período 1995 a 1998 (Figura 1) a mortalidade foi maior que o recrutamento, ocorrendo um balanço negativo, mas com valores muito próximos. $\mathrm{O}$ impacto da colheita provocou a diminuição no número de indivíduos imediatamente após esse evento, mas logo se iniciou uma recuperação da população. Este fato também foi constatado por REIS et al. (2010) na Floresta Nacional do Tapajós. Segundo RIVETT et al. (2016), após a colheita com redução de impacto, o recrutamento não é afetado de forma significativa, ao longo do tempo.

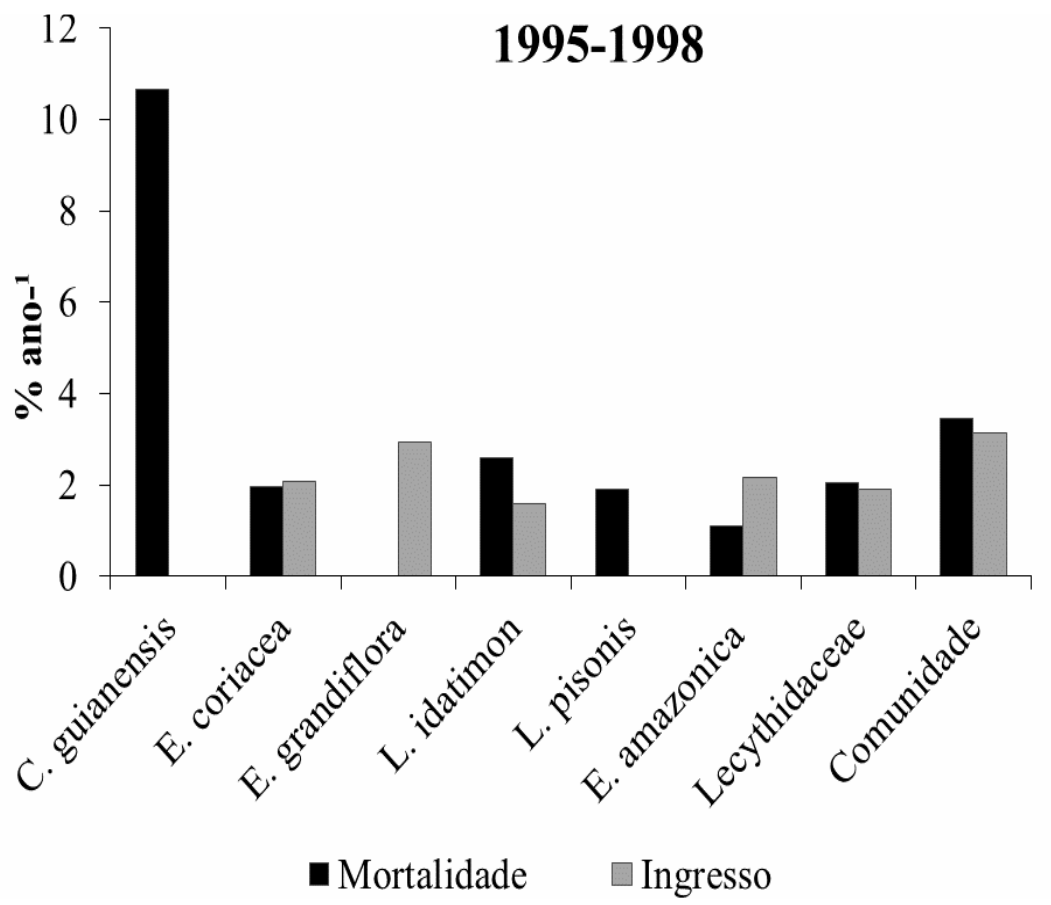

FIGURA 1. Taxas de mortalidade e recrutamento das espécies de Lecythidaceae entre 1995 a 1998, em floresta na Amazônia. 
Em resposta ao aumento da radiação solar na floresta criaram-se condições favoráveis para o recrutamento de novos indivíduos na área (DARRIGO et al., 2016). Isto pôde ser observado ao se avaliar o período pós-colheita de 1998 a 2010 (Figura 2), sendo que houve um balanço positivo na população, isto é, quando a taxa de recrutamento superou a da mortalidade (Tabela 1). Nesse período Lecythidaceae começa a se recuperar da colheita, resultando num balanço positivo ao término da avaliação em 2010 (Figura 2).

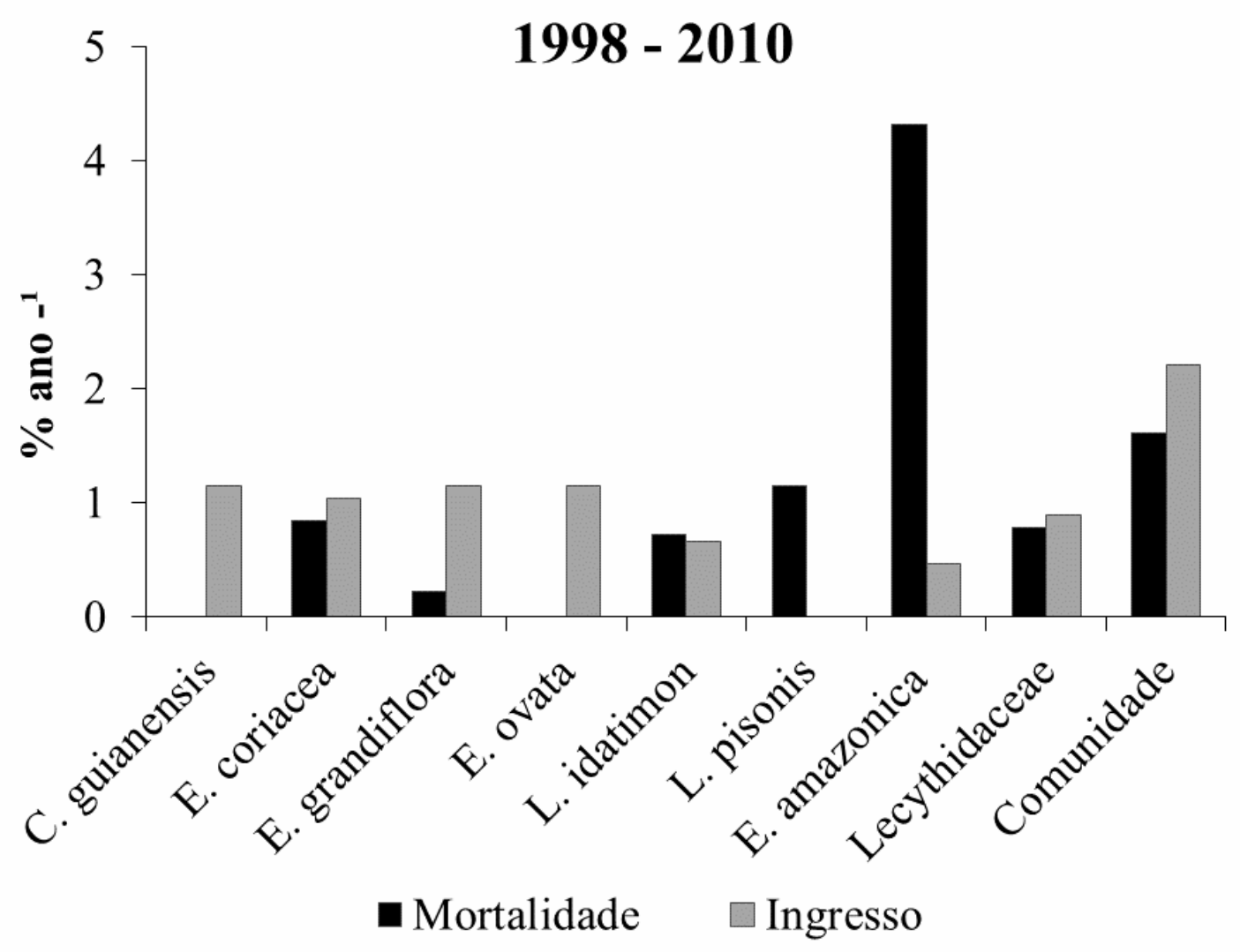

FIGURA 2. Taxas de mortalidade e recrutamento das espécies de Lecythidaceae, após a colheita florestal, de 1998 a 2010, na Amazônia.

A espécie que apresentou maior mortalidade em 15 anos de monitoramento (1995 a 2010) foi E. amazonica com taxa de $4 \%$ ao ano (Figura 3), seguida de $C$. guianensis e $L$. pisonis (Tabela 1). A mortalidade de E. amazonica foi devida a causas naturais, que foi crescente ao longo do tempo passou de 1,1\% (1995 a 1998) para $4,3 \%$ (1998 a 2010). L. pisonis teve apenas um indivíduo morto por danos na colheita, sendo o restante por mortalidade natural.

C. guianensis apresentou a segunda maior taxa de mortalidade (1995 a 2010), ocasionada por danos da colheita florestal, resultando na morte de duas árvores. Após a colheita florestal, muitas árvores são afetadas direta ou indiretamente. Os danos ocasionados diretamente podem ser quebra de copa, dos galhos, inclinação do fuste, quebra do fuste, danos no ritidoma etc. Que posteriormente podem causar a morte da árvore. Indiretamente ocorre a morte devido à mudança ambiental, como entrada de luz, e principalmente aumento da competição. 
E. grandiflora, E. coreacea e E. amazonica foram as espécies que mais se beneficiaram da abertura do dossel promovida pela colheita, em relação ao recrutamento da família (Tabela 1). A floresta tropical apresenta diferentes mosaicos de regeneração, que afetam o estabelecimento e desenvolvimento das menores árvores (FARRIOR et al., 2016), esse mosaico torna-se mais dinâmico após a colheita florestal (REIS et al., 2014).

Apesar do recrutamento inicial maior, a taxa de recrutamento foi decrescente para E. grandiflora, E. coreacea, E. amazonica e L. idetimon, passando de 2,9, 2,1, 1,6 e 2,2\% (1995 a 1998), para 1,1, 1,0, 0,7 e 0,5\% (1998 a 2010), respectivamente. Isso foi devido ao fechamento gradual do dossel. Ao contrário da espécie $E$. ovata e C. guianensis que apresentaram um aumento na taxa de recrutamento passando de nem um recrutamento no primeiro período (1995 a 1998) para 1,1\% (1998 a 2010) para ambas as espécies, demonstrando uma ecologia diferente das outras espécies da família, sendo que com maior sombreamento ocorreu mais recrutamento.

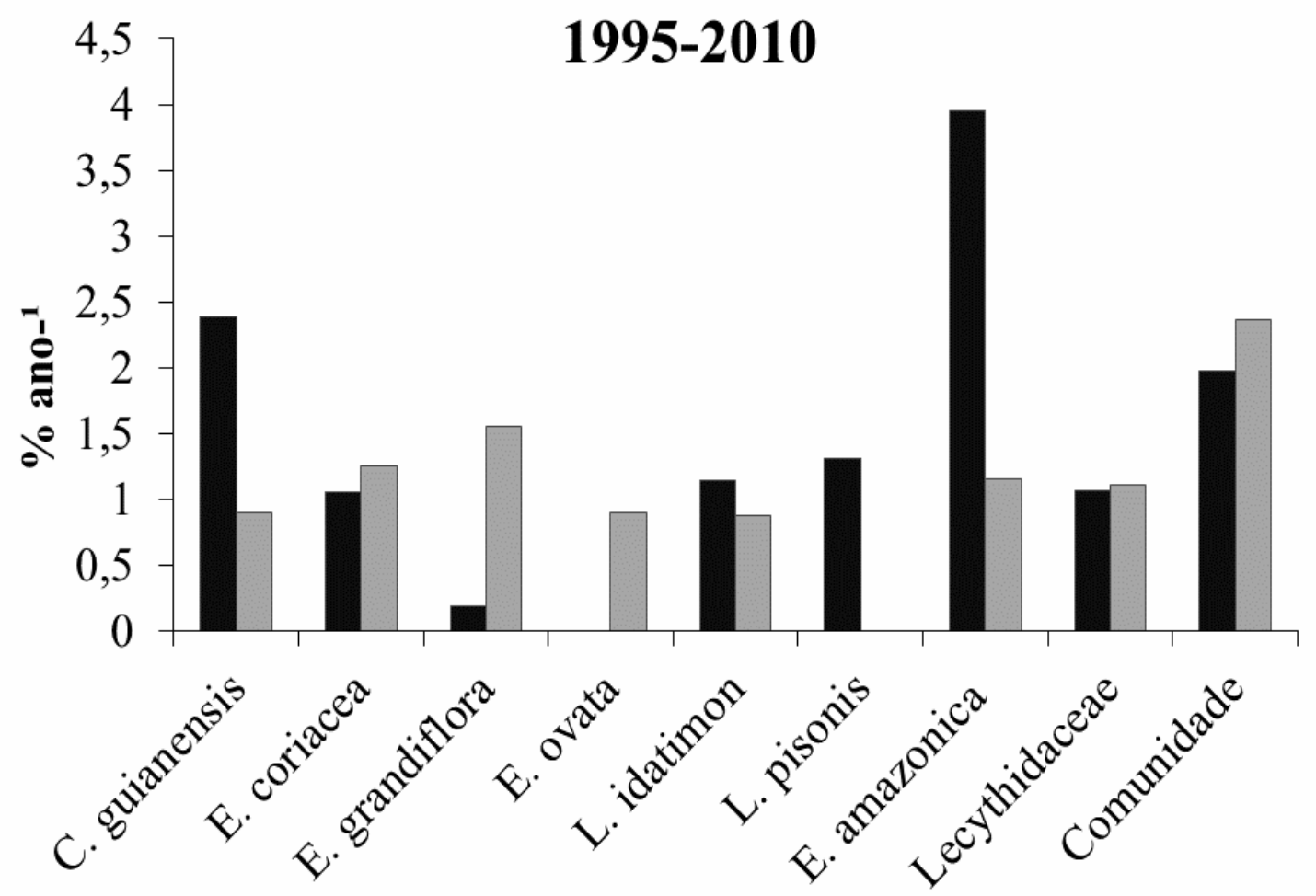

- Mortalidade $\square$ Ingresso

FIGURA 3. Taxas de mortalidade e recrutamento das espécies de Lecythidaceae de1995 a 2010, na Amazônia.

\section{CONCLUSÕES}

A colheita florestal teve impacto positivo na estrutura das populações das espécies de Lecythidaceae estudadas, que apresentou recrutamento maior que a mortalidade. A colheita também estimulou a regeneração natural e proporcionou 0 recrutamento de uma espécie não presente na população inicialmente monitorada. 


\section{AGRADECIMENTOS}

À Embrapa Amazônia Oriental pela concessão dos dados para a dissertação de mestrado que originou este artigo. À CAPES e ao CNPq pela concessão de bolsas de pós-graduação aos autores.

\section{REFERÊNCIAS}

DARRIGO, M. R.; VENTICINQUE, E. M.; SANTOS, F. A. M. Effects of reduced impact logging on the forest regeneration in the central Amazonia. Forest Ecology and Management, v. $360, \quad$ p. 52-59, 2016. Disponível em: < http://dx.doi.org/10.1016/j.foreco.2015.10.012>. doi:10.1016/j.foreco.2015.10.012

FARRIOR, C. E.; BOHLMAN, S. A.; HUBBELL, S.; PACALA, S. W. Dominance of the suppressed: Power-law size structure in tropical forests. Science, v. 351, n. 6269, p. 155-157, $2016 . \quad$ Disponível http://dx.doi.org/10.1016/10.1126/science.aad0592>. em: 10.1016/10.1126/science.aad0592

MORI, S. A.; PRANCE, G. T. Lecythidaceae - Part II - The Zygomorphic-flowered New World Genera (Couroupita, Corythophora, Bertholletia, Couratari, Eschweilera \& Lecythis), Flora Neotropica, Monograph 21, p.1-378, 1990.

NASCIMENTO, R. G. M. MACHADO, S. A.; FIGUEREDO FILHO, A.; HIGUCHI, N. Modelagem da sobrevivência, mortalidade e recrutamento de uma floresta na Amazônia Central. Scientia Forestalis, v. 41, n. 99, p. 319-330, 2013. Disponível em: <http://www.ipef.br/publicacoes/scientia/leitura. asp?Article=03\&Number=99>.

PHILLIPS, O. L.; NUNEZ, P.; TIMANA, M. E. Tree mortality and collecting botanical vouchers in tropical forests. Biotropica, v. 30, n. 2, p.298-305, 1998. Disponível em: $<$ http://dx.doi.org/10.1111/j.1744-7429.1998.tb00063.x>. doi: 10.1111/j.17447429.1998.tb00063.x

PUTZEL, L.; PETERS, C. M.; ROMO, M. Post-logging regeneration and recruitment of shihuahuaco (Dipteryx spp.) in Peruvian Amazonia: Implications for management. Forest Ecology and Management, v. 261, n. 6, p. 1099-1105, 2011. Disponível em: <http://dx.doi.org/10.1016/j.foreco.2010.12.036>. doi:10.1016/j.foreco.2010.12.036

REIS, L. P.; RUSCHEL, A. R.; COELHO, A. A.; LUZ, A. S.; MARTINS-DA-SILVA, R. C. V. Avaliação do potencial madeireiro na Floresta Nacional do Tapajós após 28 anos da exploração florestal. Pesquisa Florestal Brasileira, Colombo, v. 30, n. 64, p. 265-281, 2010. Disponível em: <http://dx.doi.org/10.4336/2010.pfb.30.64.265>. doi: 10.4336/2010.pfb.30.64.265

REIS, L. P.; RUSCHEL, A. R.; SILVA, J. N. M.; REIS, P. C. M.; CARVALHO, J. O. P.; SOARES, M. H. M. Dinâmica da distribuição diamétrica de algumas espécies de Sapotaceae após exploração florestal na Amazônia Oriental. Revista de Ciências Agrárias, v. $57, \quad$ n. 3 , p.234 - 243, 2014. Disponível em: < http://dx.doi.org/10.4322/rca.a01401>. doi: 10.4322/rca.a01401 
REIS, L. P.; SILVA, J. N. M.; REIS, P. C. M.; CARVALHO, J.O. P.; QUEIROZ, W. T.; RUSCHEL, A. R. Efeito da exploração de impacto reduzido em algumas espécies de Sapotaceae no leste da Amazônia. Floresta, v.43, n. 3, p.395 - 206, 2013. Disponível em: <http://dx.doi.org/10.5380/rf.v43i3.30808>. doi: 10.5380/rf.v43i3.30808

REIS, L. P; REIS, P. C. M.; RUSCHEL, A. R.; SILVA, J. N. M.; CARVALHO, J. O. P.; SOUZA, A. L.; SOARES, M. H. M.; MIYAHARA, R. K. N. Forest dynamics in the eastern Amazon with special reference to Sapotaceae species. Floresta, v. 45, n. 3, p.567 - 576, 2015. Disponível em: <http://dx.doi.org/10.5380/rf.v45i3.35947>. doi: 10.5380/rf.v45i3.35947

REIS, L. P.; DE CARVALHO, J. O. P.; DOS REIS, P. C. M.; GOMES, J. M.; RUSCHEL, A. R.; DA SILVA, M. G. Crescimento de mudas de Parkia gigantocarpa Ducke, em um sistema de enriquecimento em clareiras após a colheita de madeira. Ciência Florestal, v. 24, n. 2, p. 431-436, 2014 Disponível em: <http://dx.doi.org/10.5902/1980509814583>. doi:10.5902/19805098

REIS, P. C. M.; REIS, L. P.; RUSCHEL, A. R.; SILVA, J. N. M.; CARVALHO, J. O. P.; QUEIROZ, W. T. Effect of timber harvesting on density and basal area of Lecythidaceae species in the Eastern Amazon. Floresta, v. 44, n. 2, p. 229 - 238, 2014. Disponível em: <http://dx.doi.org/10.5380/rf.v44i2.33043>. doi: 10.5380/rf.v44i2.33043

REIS, P. C. M.; RUSCHEL, A. R.; REIS, L. P.; SOARES, M. H. M.; CRUZ, E. D. Lecythidaceae encontradas na floresta de Moju, Pará. Belém: Embrapa - CPATU, Documentos 416. 33 p. 2016. Disponível em: < http://ainfo.cnptia.embrapa.br/digital/bitstream/item/140791/1/DOCUMENTOS-416On-Line-AINFO.pdf >.

RIVETT, S. L.; BICKNELL, J. E.; DAVIES, Z. G. Effect of reduced-impact logging on seedling recruitment in a neotropical forest. Forest Ecology and Management, Amsterdam, n. 367, p. 71-79, 2016. Disponível em: <http://dx.doi.org/10.1016/j.foreco.2016.02.022>. doi:10.1016/j.foreco.2016.02.022

ROSSI, L. M. B.; KOEHLER, H. S.; SANQUETTA, C. R.; ARCE, J. E. Modelagem de Mortalidade em Florestas Naturais. Revista Floresta, v. 37, n. 2, p. 275-291, 2007a. Disponível em: <http://dx.doi.org/10.5380/rf.v37i2.8656>. doi: 10.5380/rf.v37i2.8656

ROSSI, L. M. B.; KOEHLER, H. S.; ARCE, J. E.; SANQUETTA, C. R. Modelagem de Recrutamento em Florestas. Floresta. Floresta, v. 37, n. 3, p. 453-467, 2007b. Disponível em: <http://dx.doi.org/10.5380/rf.v37i3.9942> doi: 10.5380/rf.v37i3.9942

SHEIL, D.; BURSLEM, D. F. R. P.; ALDER, D. The interpretation and misinterpretation of mortality rate measures. Journal of Ecology, v. 83, n. 2, p. 331333, 1995. Disponível em: <http://dx.doi.org/10.2307/2261571>. doi: $10.2307 / 2261571$ 
SHEIL, D.; MAY, R. M. Mortality and recruitment rate evaluations in heterogeneous tropical forests. Journal of Ecology, v.84, n.1, p.91-100, 1996. < http://dx.doi.org/10.2307/2261703>. doi: 10.2307/2261703

TOLEDO, J. J. DE; MAGNUSSON, W. E.; CASTILHO, C. V.; NASCIMENTO, H. E. $M$. Tree mode of death in Central Amazonia: Effects of soil and topography on tree mortality associated with storm disturbances. Forest Ecology and Management, v. 263, p. 253-261, 2012.

Disponível em: http://dx.doi.org/10.1016/j.foreco.2011.09.017>. doi:10.1016/j.foreco.2011.09.017

YAMADA, T.; HOSAKA, T.; OKUDA, T.; KASSIM, A. R. Effects of 50years of selective logging on demography of trees in a Malaysian lowland forest. Forest Ecology and Management, v. 310, p. 531-538, 2013. Disponível em: <http://dx.doi.org/10.1016/j.foreco.2013.08.057>.doi:10.1016/j.foreco.2013.08.057

ZHANG, X.; LEI, Y.; CAI, D.; LIU, F. Predicting tree recruitment with negative binomial mixture models. Forest Ecology and Management, v. 270, p. 209-215, 2012. Disponível em: < http://dx.doi.org/10.1016/j.foreco.2012.01.028>.doi: 10.1016/j.foreco.2012.01.028 